\title{
The microRNA-708-5p/ZEB1/EMT axis mediates the metastatic potential of osteosarcoma
}

\author{
TIANYU FENG ${ }^{1,2}$, ZHONGKAI ZHU $^{3}$, YAQIAN JIN ${ }^{4}$, HAO WANG $^{1}$, XIAOHAN MAO ${ }^{1}$, \\ DAN LIU ${ }^{1}$, YILING LI ${ }^{1}$, LIXIA LU ${ }^{1}$ and GUOWEI ZUO ${ }^{1}$
}

\begin{abstract}
${ }^{1}$ Key Laboratory of Diagnostic Medicine Designated by The Chinese Ministry of Education, Department of Laboratory Medicine, Chongqing Medical University, Chongqing 400016; ${ }^{2}$ Laboratory of Experimental Oncology, State Key Laboratory of Biotherapy and Cancer Center, West China Hospital, Sichuan University, and Collaborative Innovation Center, Chengdu, Sichuan 610041; ${ }^{3}$ Department of Cardiology, The First Affiliated Hospital of Chongqing Medical University;

${ }^{4}$ Chongqing Key Laboratory of Traditional Chinese Medicine for Prevention and Cure of Metabolic Diseases, College of Traditional Chinese Medicine, Chongqing Medical University, Chongqing 400016, P.R. China
\end{abstract}

Received July 5, 2019; Accepted November 22, 2019

DOI: $10.3892 /$ or.2019.7452

\begin{abstract}
MicroRNA-708-5p (miR-708-5p) and epithelialto-mesenchymal transition (EMT) have been widely identified to contribute to the pathogenesis and progression of multiple cancers. However, the connection between miR-708-5p and EMT has not been sufficiently clarified. Therefore, our research aimed to investigate the impact of miR-708-5p on EMT and the metastasis of osteosarcoma (OS). We first analyzed the differentially expressed microRNAs (DEmiRNAs) from the GSE70367 dataset. We found that the expression of miR-708-5p was lower in OS cells. Overexpression of miR-708-5p was able to impair the migration and invasion of OS cells. Moreover, miR-708-5p inhibited EMT of OS cells MG63 and SaOS-2, wherein E-cadherin was increased, and $\mathrm{N}$-cadherin, vimentin, and Snail were decreased. Semaphorin 4C (SEMA4C), mitogen-activated protein kinase kinase kinase 3 (MAP3K3), and zinc finger E-box-binding homeobox 1 (ZEBI) were predicted as target genes of miR-708-5p by bioinformatics method. Only $Z E B 1$, one of the EMT-inducing transcription factors, was validated as the direct target gene of miR-708-5p in OS cells through dual-luciferase reporter assay and Western blot analysis. Knockdown of ZEB1 was found to inhibit the metastasis of MG63 and SaOS-2 cells, whereas ZEB1 overexpression promoted their metastasis. In summary, miR-708-5p impaired the metastasis and EMT of OS, which was found to be mediated by inhibition of ZEB1.
\end{abstract}

Correspondence to: Dr Guowei Zuo, Key Laboratory of Diagnostic Medicine Designated by The Chinese Ministry of Education, Department of Laboratory Medicine, Chongqing Medical University, Chongqing 400016, P.R. China

E-mail: zuoguowei@cqmu.edu.cn

Key words: microRNA-708-5p, osteosarcoma, epithelial-tomesenchymal transition, metastasis

\section{Introduction}

Osteosarcoma (OS) is a primitive mesenchymal cell-derived bone tumor that most commonly occurs at the metaphyses of long bones in adolescents (1). Although the annual incidence of OS is three to four patients per million (2), OS is ranked among the leading causes of cancer-related deaths in the pediatric age group (3). OS is rarely cured because it spreads systemically, with the lung being the most common metastatic hotspot followed by bone (4). The prognosis of OS patients has greatly improved due to multi-agent chemotherapy, with long-term survival rates improving from less than $20 \%$ to $65-70 \%$ (5). Aside from low prevalence and easy metastasis, large tumor heterogeneity causes difficulty in significantly improving the survival rates of patients (3). Therefore, there is an urgent research aim to decipher the molecular mechanisms underlying the occurrence and development of OS, and explore possible effective molecular treatment strategies to halt its metastasis.

MicroRNAs (miRNAs) are endogenous $22 \mathrm{nt}$, short, non-coding RNAs that regulate gene expression through direct post-transcriptional repression of mRNA targets $(6,7)$. Since the discovery of the first miRNA, lin-4, an increasing number of miRNAs have been found, and a large number of target genes have been validated. Mammalian miRNAs have been identified to play multiple roles in diverse cellular (including cell death and cell proliferation) and physiological processes $(7,8)$. In the field of cancer, miRNAs function as oncogenes or tumor suppressors through their participation in tumor growth, invasion, angiogenesis, and immune evasion (9). Although the molecular etiology of OS remains to be further defined, the effects of miRNAs on the pathogenesis and progression of OS have been widely investigated (10). A previous study found that miR-708-5p was downregulated in OS samples compared with non-neoplastic bone samples (11). In our bioinformatics analysis, we detected that miR-708-5p was decreased in OS cells MG63 and SaOS-2 compared with human bone marrow-derived mesenchymal stem cells 
(hMSCs). The differential expression of miR-708-5p between normal and OS cells impelled us to clarify the specific function and mechanism of miR-708-5p in OS.

Epithelial cells converting to mesenchymal cells is the basic process for embryonic development. This process consists of important phenotypic changes, which include loss of cell-cell adhesion, lack of cell polarity, and development of migratory and invasive properties (12). Carcinoma cells are able to acquire increased motility and impaired intercellular adhesion to develop mesenchymal cell morphology and infiltrate ambient tissues through epithelial-to-mesenchymal transition (EMT) (13). EMT transcription factors (EMT-TFs), such as Snail1, Twist1, ZEB1 and ZEB2, activate EMT $(14,15)$. EMT-TFs act as repressors of E-cadherin genes by directly (Snail1, ZEB1 and ZEB2) or indirectly (Twist1) binding to the E-boxes on the E-cadherin promoter to repress its transcription. Moreover, EMT can be regulated by TGF- $\beta$, hypoxia, Notch, and WNT pathways. MicroRNAs such as miR-200, miR-103/107, and miR-181a can mediate EMT by targeting EMT-TFs $(14,15)$. However, the relationship between miR-708-5p and EMT in OS has been insufficiently clarified. Therefore, we investigated the specific impacts of miR-708-5p on the metastasis of OS and EMT and explored its target genes in OS.

Zinc finger E-box-binding homeobox 1 (ZEB1) and its mammalian paralog ZEB2 belong to the ZEB family within the ZF (zinc finger) class of homeodomain transcription factors. The ZEB family plays an important role in normal embryonic development, including inducing EMT, in which epithelial cells lose polarity and obtain invasive properties, thus becoming mesenchymal cells (16). The main role of ZEB1 during EMT is the inhibition of the expression of E-cadherin by binding to its promoter region, which results in the cells losing their epithelial properties (17). ZEB1 has been reported to be associated with proliferation, apoptosis, migration, invasion, and EMT of OS (18-21). ZEB1 has been validated to be regulated by several miRNAs in OS. miR-126 was testified to inhibit proliferation, migration, invasion, and EMT in OS via targeting ZEB1 (19), miR-130a was reported to inhibit OS growth and metastasis through directly targeting ZEB1 (20). Nonetheless, the relationship between miR-708-5p and ZEB1 has not been fully explored in OS.

In the present study, we examined the expression of miR-708-5p in OS cell lines and reintroduced miR-708-5p in OS cell lines utilizing cell transfection. The effects of miR-708-5p on OS cell migration and invasion were observed, the direct target gene of miR-708-5p was validated, and the EMT of OS cells was explored. The results will provide novel theoretical strategies for the diagnosis and treatment of OS. In summary, this study will help us further decipher the molecular etiology of OS.

\section{Materials and methods}

Cell lines and human bone marrow mesenchymal stem cells (hMSCs). 293T and human OS cell lines MG63, SaOS-2 and normal bone marrow cell line HS-5 were purchased from the American Type Culture Collection (Manassas, VA, USA). Upon approval by the Ethics Committee of the Children's Hospital of Chongqing Medical University (Chongqing,
China), human bone marrow-derived mesenchymal stem cells (hMSCs) were obtained from three healthy donors who provided informed consent. hMSCs were isolated from bone marrow utilizing density gradient centrifugation as previous described $(22,23)$. In brief, Ficoll medium (TBD Science) and bone marrow dilution solution (diluted with PBS of 1:1 ratio) were gently added into a centrifuge tube at a 1:2 ratio and then centrifuged for $30 \mathrm{~min}$ at $644 \mathrm{x} \mathrm{g}$. The cloudiness interface layer which contains monocytes were aspirated into another centrifuge tube and mixed with PBS, and the mixture was centrifuged for $3 \mathrm{~min}$ at $161 \mathrm{x}$ g. The supernatant of the mixture was discarded, and then complete culture medium was added to suspend the primary cells. The primary cell suspension was transferred onto T-25 flasks, and the culture medium was discarded to abandon unattached cells. All the above cells were maintained in Dulbecco's modified Eagle's medium (Hyclone, GE Healthcare) supplemented with $10 \%$ fetal bovine serum (FBS; Lonsera) and $100 \mathrm{U} / \mathrm{ml}$ streptomycin/penicillin at $37^{\circ} \mathrm{C}$ in $5 \% \mathrm{CO}_{2}$.

Differentially expressed miRNAs. miRNA Expression data from human osteosarcoma (OS) dataset GSE70367 was downloaded from the National Center for Biotechnology Information (NCBI) Gene Expression Omnibus (GEO) database (http://www.ncbi. nlm.nih.gov/geo). The dataset GSE70376 was based on GPL16384 platform. GSE70376 dataset includes miRNAs expression data in five OS cell lines (MG63, Saos, HOS, NY and Hu09) and human mesenchymal stem cells (hMSCs).

Cell transfection. miRNA mimics, scramble negative control mimics (scramble NC mimic) and ZEB1-specific small interfering RNA (si-ZEB1) were synthesized by GenePharma (Shanghai, China). The ZEB1 overexpression plasmid pEZ-ZEB1 and empty plasmid pEZ-M35 were purchased from GeneCopoeia. MicroRNA-708-5p mimic (20 nM) and scramble NC mimic $(20 \mathrm{nM})$ were transfected into OS cells using Lipofectamine 2000 (Invitrogen; Thermo Fisher Scientific, Inc.) according to the manufacturer's instructions. The subsequent experiments were performed after the cells were transfected for $48 \mathrm{~h}$.

$R N A$ extraction and $R T-q P C R$. Total RNA was extracted using TRIzol reagent (Tiangen) in line with the manufacturer's instructions and first-strand DNA was synthesized using the Reverse Transcriptase (RT) M-MLV kit with random hexamer primers (Takara). miRNAs were extracted utilizing Magen Hipure Universal RNA Kits (Magen, Guangzhou, China) according to manufacturer's instructions. Target-specific primers were used for reverse transcription [RT primers (5'-3') for miR-708-5p: GTCGTATCCAGTGCAGGGTCCGAG GTATTCGCACTGGATACGACCCCAGC and RT primers (5'-3') for U6: GTCGTATCCAGTGCAGGGTCCGAGGTA TTCGCACTGGATACGACTTGCGC]. TB Green ${ }^{\mathrm{TM}}$ Premix Ex $\operatorname{Taq}^{T M}$ II (Takara) was used to determine the expression of miRNAs and mRNAs utilizing the $2^{-\Delta \Delta C q}$ (24) method by CFX Connect Real-Time PCR system (Bio-Rad Laboratories, Inc). GAPDH and U6 were used as the internal references for mRNAs and miRNAs, respectively. The sequence of primers for RT-qPCR are listed in Table I. 
Wound healing assay. To determine whether miR-708-5p affects the lateral migration ability of OS cells, cells were digested and resuspended in DMEM after $48 \mathrm{~h}$ of transfection, and then plated into 6 -well plates. When the cell confluence reached $80-90 \%$, a straight scratch was made using a $10-\mu 1$ pipette tip. The floating debris were removed by washing with PBS and the culture medium was replaced with serum-free DMEM culture media. The final photomicrographs were captured utilizing an inverted phase contrast microscope (Nikon, Japan) at a magnification of x100 at 24 h for MG63 cells or $48 \mathrm{~h}$ for $\mathrm{SaOS}-2$ cells.

Cell migration and invasion assays. For the cell migration assay, $1.5 \times 10^{4} \mathrm{OS}$ cells were plated into 8 -micron inserts in a 24-well plate containing serum-free DMEM. For the invasion assay, the Transwell chambers were pre-coated with 1:3 diluted Matrigel (Solarbio) and $3 \times 10^{4}$ OS cells were plated into 8-micron inserts. The lower chambers were filled with $700 \mu 1$ complete medium with $10 \%$ FBS. Cells were allowed to migrate for $24 \mathrm{~h}$ (MG63) or $48 \mathrm{~h}$ (SaOS-2). After migration, the inserts were washed twice with fresh PBS and fixed with $4 \%$ paraformaldehyde for $10 \mathrm{~min}$. After fixation, the inserts were washed with $\mathrm{ddH}_{2} \mathrm{O}$ twice and stained with $5 \%$ crystal violet solution for $15 \mathrm{~min}$. After staining, the inserts were washed with $\mathrm{ddH}_{2} \mathrm{O}$ and the non-migrated cells were carefully removed with a cotton swap. The migrated cells were captured and counted at x100 magnification under an inverted phase contrast microscope (Nikon, Japan) in at least five visual fields.

Western blot analysis. Total protein was extracted after transfection for at least $48 \mathrm{~h}$. OS cells were lysed with RIPA buffer containing protease inhibitors and phosphatase inhibitor (Roche Applied Science), and then centrifuged at $12,000 \mathrm{x}$ g for $25 \mathrm{~min}$ at $4^{\circ} \mathrm{C}$ and the supernatants were collected. Concentration of protein was measured by BCA assay and then the protein was boiled for 10 min in loading buffer. Equal amounts $(15 \mathrm{ng})$ of cell protein were loaded onto $8-10 \%$ SDS-PAGE gels and subsequently transferred onto polyvinylidene difluoride membranes (PVDF; Millipore) membranes. The membranes were blocked with $5 \%$ bovine serum albumin (BSA; Solarbio) diluted with TBST and then incubated with primary antibodies overnight at $4^{\circ} \mathrm{C}$. Western blotting was performed utilizing primary antibodies specific for $\beta$-actin (1:1,000 dilution; cat. no. TA-09; Zhongshan Golden Bridge Biotechnology), MMP2 (1:1,000 dilution; D8N9Y; Cell Signaling Technology), MMP7 (1:1,000 dilution; AF0218; Affinity Biosciences), MMP9 (1:1,000 dilution; AF5228; Affinity Biosciences), E-cadherin (1:500 dilution; sc-21791; Santa Cruz Biotechnology, Inc.), N-cadherin (1:500 dilution; sc-53488; Santa Cruz Biotechnology, Inc.), Vimentin (1:500 dilution; WL01960; Wanleibio), ZEB1 (1:1,000 dilution; YN3011; Immunoway) and Snail (1:1,000 dilution; WL01863; Wanleibio). After incubation with the primary antibody at $4^{\circ} \mathrm{C}$ overnight, the membranes were washed with TBST for $30 \mathrm{~min}$, and then the membranes were incubated with HRP-labelled IgG secondary antibody (1:5,000 dilution; Zhongshan Goldenbridge Biotechnology) at $37^{\circ} \mathrm{C}$ for $1 \mathrm{~h}$. The protein bands were detected using ECL solution kit (Immobilon Western, Millipore). The protein bands were quantified using Image Lab software version 5.2 (Bio-Rad
Table I. Primer sequences used for RT-qPCR.

Genes $\quad$ Sequences (5'-3')

miR-708-5p

Forward

GGCGCGAAGGAGCTTACAATCTA

Reverse

ATCCAGTGCAGGGTCCGAGG

U6

Forward GTGCTCGCTTCGGCAGCACA

Reverse ATCCAGTGCAGGGTCCGAGG

GAPDH

Forward

CAGCGACACCCACTCCTC

Reverse TGAGGTCCACCACCCTGT

MMP2

Forward

AGACATACATCTTTGCTGGAGACA

Reverse

CTTGAAGAAGTAGCTGTGACCG

MMP7

Forward

GGAGGAGATGCTCACTTCGAT

Reverse

AGGAATGTCCCATACCCAAAGA

MMP9

Forward

GGGACGCAGACATCGTCATC

Reverse

TCGTCATCGTCGAAATGGGC

E-cadherin

Forward

AATGAAGCCCCCATCTTTG

Reverse

CAGCCAGTTGGCAGTGTCT

$N$-cadherin

Forward

CCATCAAGCCTGTGGGAATC

Reverse

GCCGCTTTAAGGCCCTCAT

Vimentin

Forward

TGCTCAATGTTAAGATGGCCCT

Reverse

TTCAAGGTCATCGTGATGCTGA

Snail

Forward CCATGTCCGGACCCACAC

Reverse GCCGGACTCTTGGTGCTT

ZEB1

Forward CCAAGCTTATGAAAGTTACAAATTATAA

Reverse CGGGATCCCTTCAAAGGACTTTGTAGAT

Laboratories, Inc.), and the values are expressed relative to $\beta$-actin.

Dual-luciferase reporter assay. We cloned the sequences from miR-708-5p binding sites on 3' UTRs of ZEB1, SEMAC and MAP3K3, respectively, into the pGL6-miR (Beyotime Institute of Biotechnology) reporter plasmid. The miRNA-mRNA binding sites were also mutated and cloned into the reporter plasmid. When the $293 \mathrm{~T}$ cells reached $70 \%$ confluence in 24-well plates, the cells were co-transfected with $400 \mathrm{ng}$ wild-type (or mutant) reporter plasmids plus miR-708-5p mimic (or scramble NC mimic), together with 10 ng Renilla reporter plasmid PRL-SV40 per well, and the mixture was diluted in serum-free medium. The medium was replaced with complete medium containing $10 \%$ FBS after $6 \mathrm{~h}$, and the 293T cells were lysed to be measured $48 \mathrm{~h}$ 
post-transfection. Luciferase activity assay was then performed using the Dual-Luciferase Reporter Assay System (Promega), and normalized with the Renilla activity.

Immunofluorescence. Cells cultured on crawling pieces were washed with PBS and fixed with $4 \%$ paraformaldehyde for $20 \mathrm{~min}$, and $0.25 \%$ Triton X-100 (Solarbio) was used for permeabilization at $37^{\circ} \mathrm{C}$ for $15 \mathrm{~min}$. Next, $0.5 \%$ Triton X-100 was used for permeabilizing at room temperature for $20 \mathrm{~min}$. Goat serum (AR0009, Boster Biological Technology) was used for blocking at $37^{\circ} \mathrm{C}$ for $30 \mathrm{~min}$ and then the cells were incubated with primary antibodies specific for ZEB1 (1:200 dilution; sc-515797; Santa Cruz Biotechnology, Inc.) at $4^{\circ} \mathrm{C}$ overnight. Then the crawling pieces were washed with PBS for 3 times and Rhodamine (TRITC)-conjugated goat anti-mouse IgG (ZF0313; Zhongshan Goldenbridge Biotechnology, Beijing, China) was used to incubate the cells at $37^{\circ} \mathrm{C}$ for $1 \mathrm{~h}$. Nuclei were stained with Hoechst 33258 (DA0011, Leagene Biotechnology) at room temperature for $5 \mathrm{~min}$. Images were captured using a fluorescence microscope at $\mathrm{x} 400$ magnification (DM4B, Leica, Germany). ImageJ software (version 1.48; National Institutes of Health, Bethesda, MD, USA) was utilized to quantify the cell fluorescence.

Statistical analysis. GraphPad Prism 6 (GraphPad Software, Inc.) was utilized to analyze the data. All the data are presented as the mean \pm standard deviation from at least three independent experiments. Unpaired t-test was used to compare two groups. Comparisons between multiple groups (when $>2$ groups) were performed by one-way analysis of variance and Tukey's multiple comparision test in which pairwise comparisons between all groups were performed. Statistical significance was set at $\mathrm{P}<0.05$.

\section{Results}

Lower expression of miR-708-5p in OS cell lines. The microRNA expression data were obtained from Gene Expression Omnibus (GEO) database (http://www.ncbi. nlm.nih.gov/geo) (accession number: GSE70367), which partly consists of five OS cell lines (MG63, Saos-2, Hu09, NY, HOS) and normal control cells (hMSCs). These data were analyzed using GEO2R (https://www.ncbi.nlm.nih. gov/geo/geo2r/), the parameter was set as default. The top 50 differentially expressed miRNAs are presented in a heatmap drawn using HemI (http://hemi.biocuckoo.org/), in which hsa-miR-708-5p was decreased in the OS cell lines (MG63, SaOS-2, Hu09, NY, HOS) compared with that noted in the human bone marrow-derived mesenchymal stem cells (hMSCs) (Fig. 1A). Furthermore, the expression of miR-708-5p was significantly lower in the MG63 and SaOS-2 cells in comparison with the hMSCs and HS-5, as validated by RT-qPCR (Fig. 1B-E). Moreover, miR-708-5p was reported to be downregulated in OS samples and was correlated with OS prognosis and progression (3). These findings suggest that miR-708-5p may serve as a therapeutic miRNA for OS.

Overexpression of miR-708-5p impairs the migration and invasion of OS cells. MicroRNA-708-5p mimic (708-5p mimic) and scramble $\mathrm{NC}$ mimic (NC mimic) were transfected into OS MG63 and SaOS-2 cells and the transfection efficiency was verified (Fig. 1F and G). MG63 and SaOS-2 cells transfected with 708-5p mimic showed significantly increased miR-708-5p mRNA expression. To investigate whether miR-708-5p affects the migration and invasion of OS, wound healing and Transwell assays were conducted. The wound healing rate of MG63 (24 h) and SaOS-2 (48 h) was significantly decreased in the 708-5p mimic group compared with the NC mimic group (Fig. 2A and B). Concordant with the wound healing assay, the numbers of transmembrane migratory and invasive cells were significantly decreased in the 708-5p mimic group compared with the NC mimic group in the MG63 and SaOS-2 cells (Fig. 2C and D).

Given that matrix metalloproteinases (MMPs) play a crucial role during tumor metastasis, we also assessed the mRNA (Fig. 3A and B) and protein levels of MMP2, MMP7, and MMP9 (Fig. 3C-E) in the MG63 and SaOS-2 cells. The mRNA and protein levels of MMP2, MMP7, and MMP9 were significantly decreased after miR-708-5p was overexpressed in MG63 cells (Fig. 3D). Likewise, the mRNA and protein levels of MMP2 and MMP9 were significantly decreased after miR-708-5p was overexpressed in the SaOS-2 (Fig. 3E).

miR-708-5p suppresses epithelial-to-mesenchymal transition (EMT) of OS cells. To further investigate whether miR-708-5p affects EMT in OS, we detected the mRNA and protein levels of EMT-related biomarkers. As shown in Fig. 3, miR-708-5p overexpression significantly induced the expression of epithelial biomarker E-cadherin while significantly repressing mesenchymal biomarkers $\mathrm{N}$-cadherin, vimentin, and EMT-related transcription factor Snail. These results indicate that miR-708-5p impairs the EMT of OS cells.

Zinc finger E-box-binding homeobox 1 (ZEB1) is a direct target gene of miR-708-5p. To explore the miR-708-5p-mediated downstream regulator of OS cells, TargetScan7.2 (http://www.targetscan.org/vert_72/) was applied to predict the potential target genes of miR-708-5p. Semaphorin 4C $(S E M A 4 C)$, mitogen-activated protein kinase kinase kinase 3 (MAP3K3), and ZEBI are types of tumor metastasis-associated genes and were thus screened as our candidate target genes of miR-708-5p. To validate whether miR-708-5p binds to the $3^{\prime}$ UTR (3' untranslated region) of these candidate genes, we cloned a part of 3'UTR (containing the binding sites) of candidate genes into pLG6-miR to construct a dual-luciferase reporter system. 3'UTR luciferase reporter plasmid (wild-type; WT) or 3'UTR mutated (MUT) luciferase reporter plasmid (mutant type) with microRNA mimic or NC mimic were co-transfected into $293 \mathrm{~T}$ cells. miR-708-5p overexpression significantly decreased the luciferase activities in the wild-type 3'UTR of SEMA4C, MAP3K3, and ZEB1, whereas the mutant type exhibited no significant changes (Fig. 4A-C). Nonetheless, only the protein level of ZEB1 was decreased after miR-708-5p overexpression in MG63 and SaOS-2 cells (Fig. 4D). This finding indicates that ZEB1 was directly targeted by miR-708-5p in OS. Moreover, immunofluorescence results showed that miR-708-5p overexpression could inhibit the fluorescence intensity of ZEB1 in MG63 cells (Fig. 4E). 


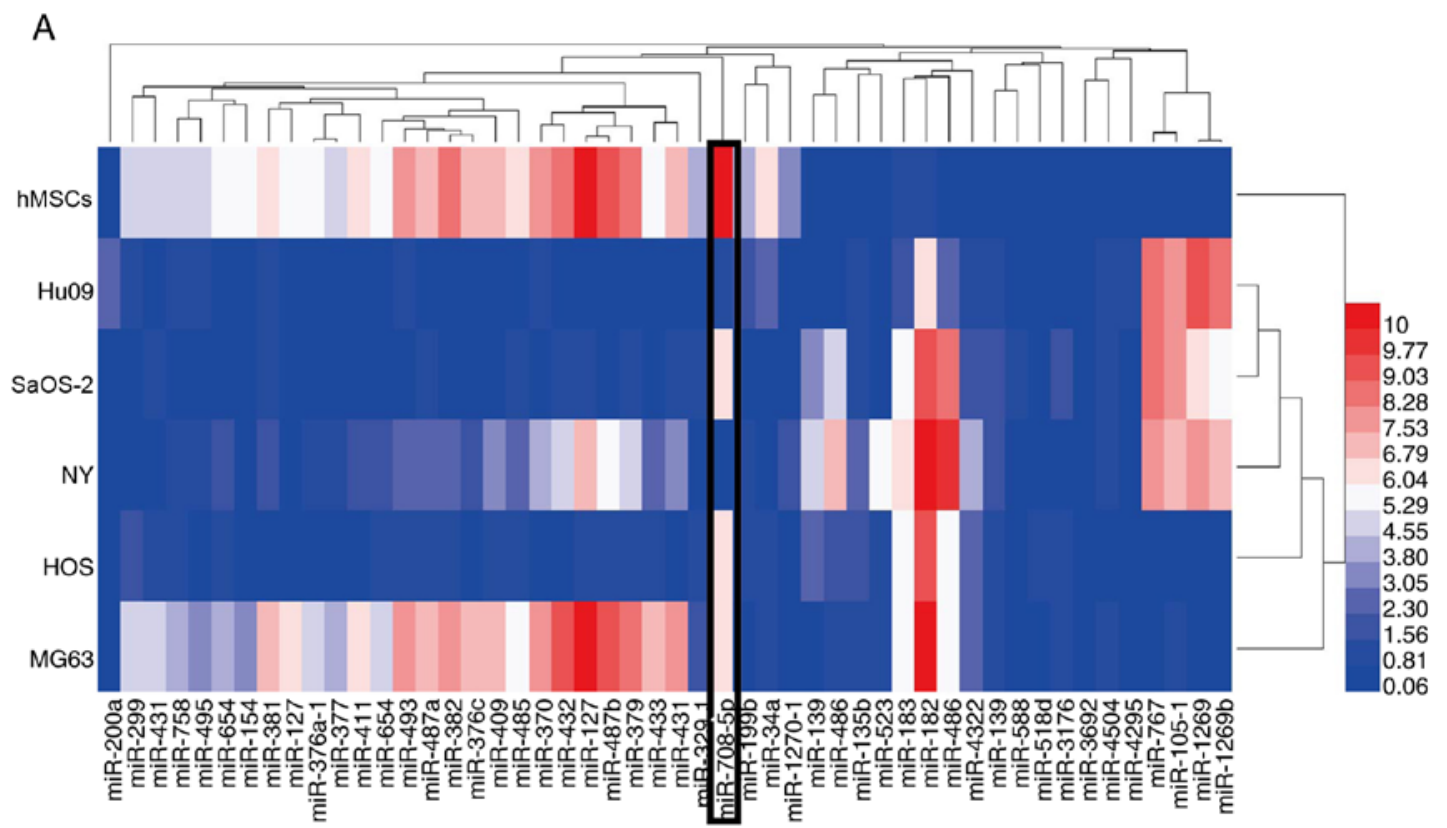

B

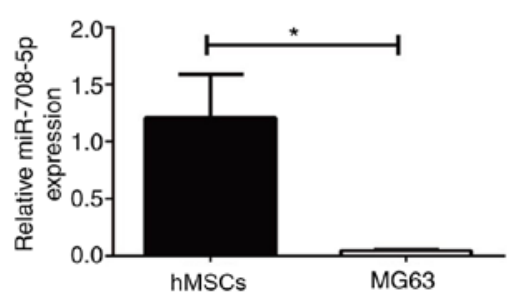

D
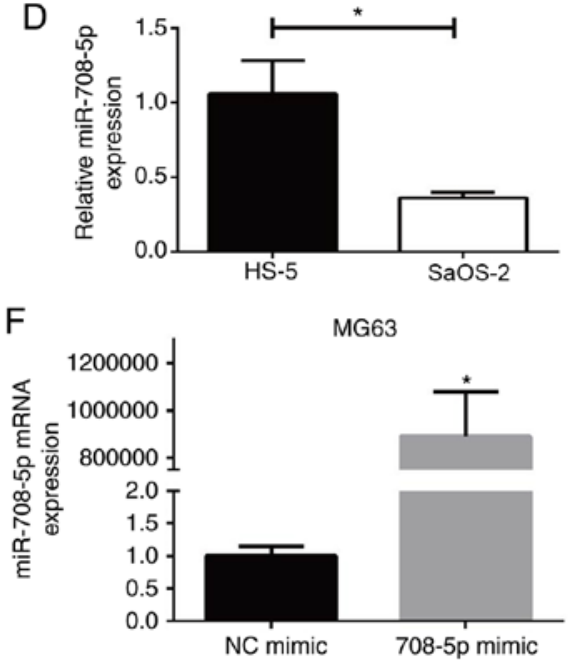

C

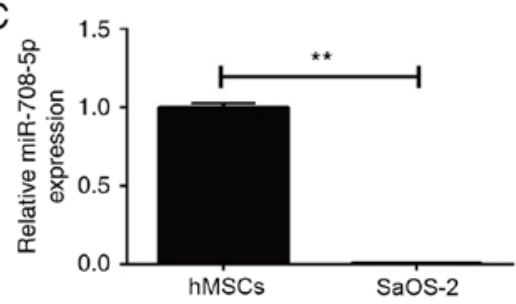

E

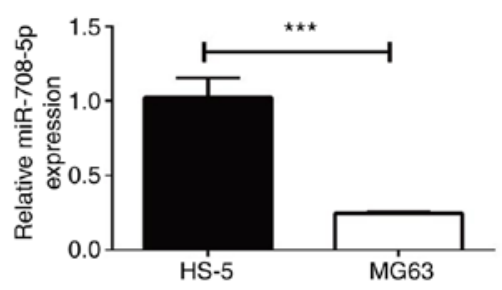

$\mathrm{G}$

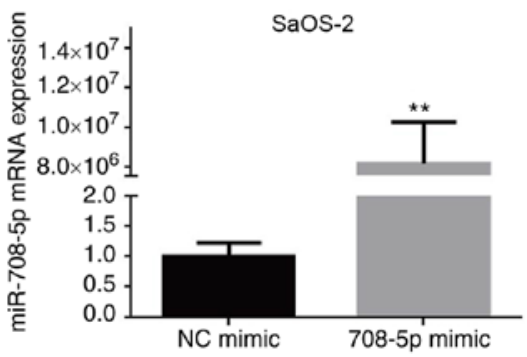

Figure 1. Differentially expressed miRNAs in OS cell lines and miR-708-5p expression in OS cell lines. (A) Top 50 differentially expressed miRNAs in OS cell lines (MG63, HOS, NY, SaOS-2, Hu09) and normal hMSCs. (B-E) Expression of miR-708-5p in MG63, SaOS-2, hMSC and HS-5 cells by RT-qPCR. All data are presented as mean \pm SD from at least three independent experiments. ${ }^{*} \mathrm{P}<0.05,{ }^{* * *} \mathrm{P}<0.01,{ }^{* * * *} \mathrm{P}<0.001$ vs. hMSCs or HS-5 cells. (F and G) Expression of miR-708-5p in MG63 and SaOS-2 cells after transfection with $20 \mathrm{nM}$ miR-708-5p mimics (708-5p mimics) or the scramble negative control (NC mimic). All data are presented as mean $\pm \mathrm{SD}$ from at least three independent experiments. ${ }^{*} \mathrm{P}<0.05,{ }^{* *} \mathrm{P}<0.01$ vs. the NC mimic. OS, osteosarcoma; hMSCs, human bone marrow-derived mesenchymal stem cells.

ZEB1 is upregulated in OS and suppression of ZEB1 inhibits OS cell migration and invasion. We downloaded the expression box plot of ZEB1 in sarcoma tissues and normal tissues from the Gene Expression Profiling Interactive Analysis database (GEPIA), a web server for cancer and normal gene expression profiling and interactive analyses. As shown in Fig. 5A, ZEB1 was upregulated in sarcoma tissues when compared to that in normal tissues. Hence, we further assessed the protein levels of ZEB1 in OS MG63 and SaOS-2 cells. We found that ZEB1 was upregulated in MG63 and SaOS-2 cells compared with that noted in the hMSCs and HS-5 (Fig. 5B). These findings suggest that ZEB1 may act as an oncogene in OS. To better elucidate the role of ZEB1 in the metastasis of OS, ZEB1 was inhibited through small-interference RNA (si-ZEB1). ZEB1 mRNA and protein expression levels were significantly decreased after transfection with siRNA, as determined by RT-qPCR and 
A
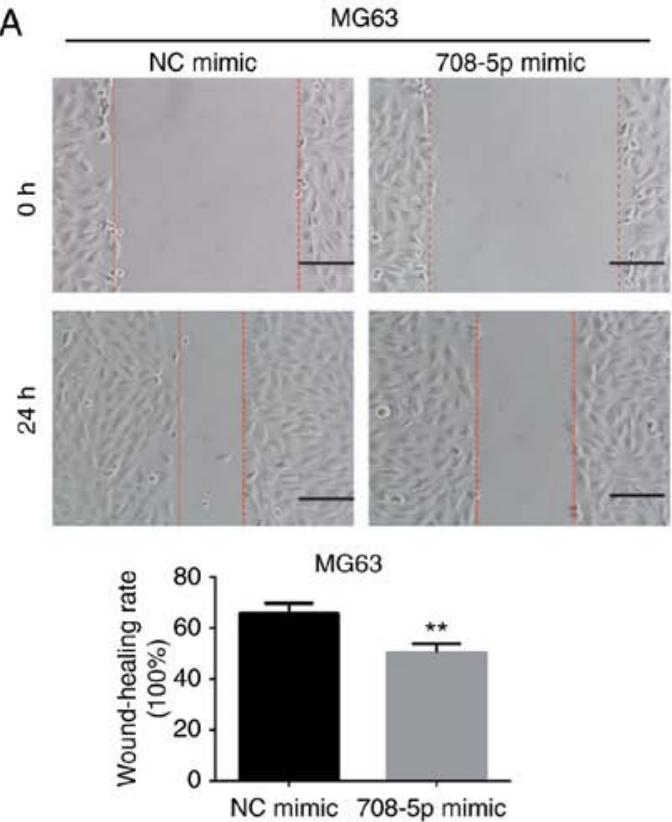

C
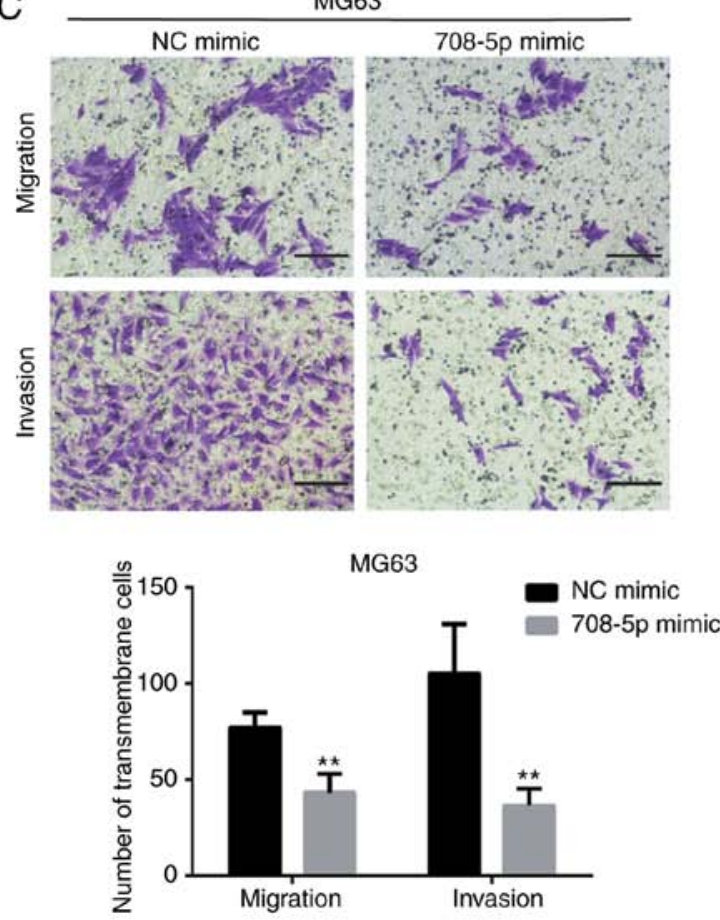

B
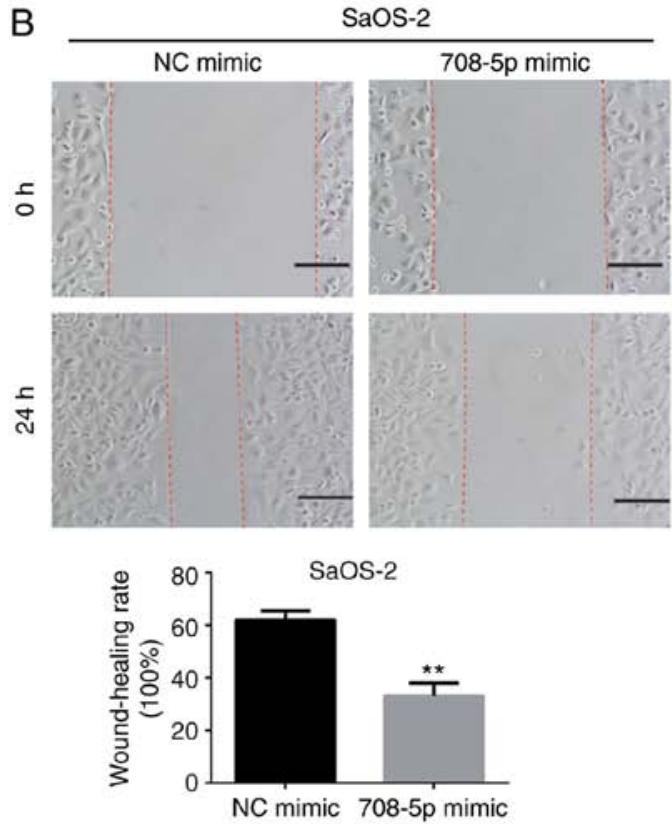

D
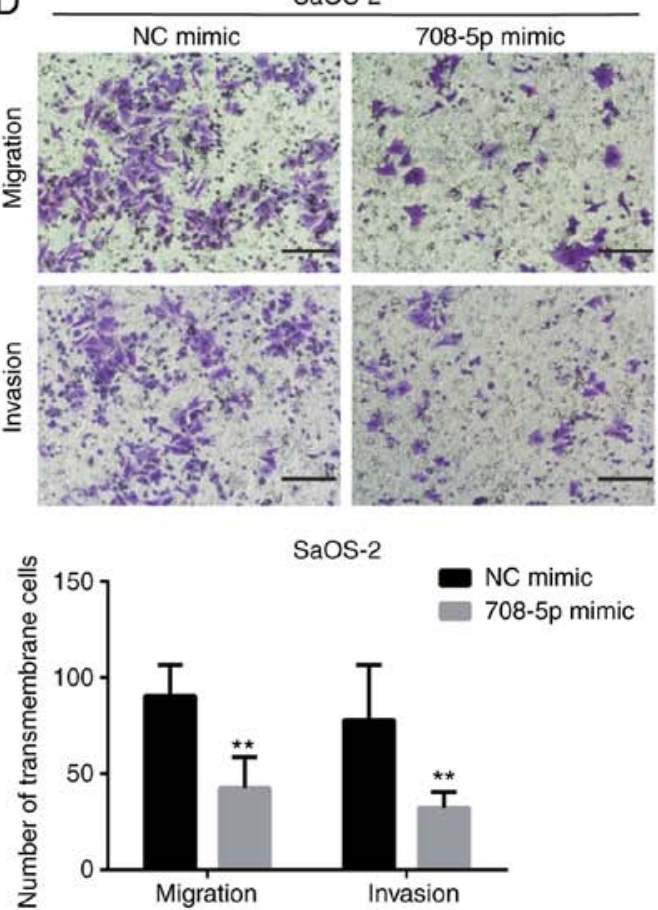

Figure 2. miR-708-5p expression suppressed the migration and invasion of OS cells. Effects of miR-708-5p on the lateral migration of (A) MG63 and (B) SaOS-2 cells and on the longitudinal migration and invasion of (C) MG63 and (D) SaOS-2 cells following transfection of the miR-708-5p mimics (708-5p mimics) or the scramble negative control ( $\mathrm{NC}$ mimic). All data are presented as mean $\pm \mathrm{SD}$ from at least three independent experiments. ${ }^{* * *} \mathrm{P}<0.01, \mathrm{NC}$ mimic vs. 708-5p mimic. OS, osteosarcoma.

Western blot analysis (Fig. 5C and E). Moreover, the numbers of invasive and migratory (transmembrane) cells were decreased after ZEB1 was effectively knocked down (Fig. 5D and F).

ZEB1 reverses the miR-708-5p-mediated suppression of cell metastasis. To further confirm whether ZEB1 plays a vital role in the miR-708-5p-mediated suppression of cell migration and invasion, rescue experiments were conducted. As shown in Fig. 6A and C, the mRNA and protein expression levels of ZEB1 were increased after transfected with pEZ-ZEB1 in MG63 and $\mathrm{SaOS}-2$ cell lines. Subsequently, the numbers of invasive and migratory (transmembrane) cells were increased after ZEB1 was reintroduced (Fig. 6B, D and E). In brief, ZEB1 abrogated the miR-708-5p-mediated inhibition of metastasis of OS cells.

\section{Discussion}

Currently, miRNAs are considered to regulate more than $60 \%$ of human protein-coding genes (25). In addition, miRNAs generally bind to the 3'-untranslated regions (3'UTR) of target mRNA genes functioning in two patterns: Endonucleolytic cleavage and translational repression or deadenylation (26). 

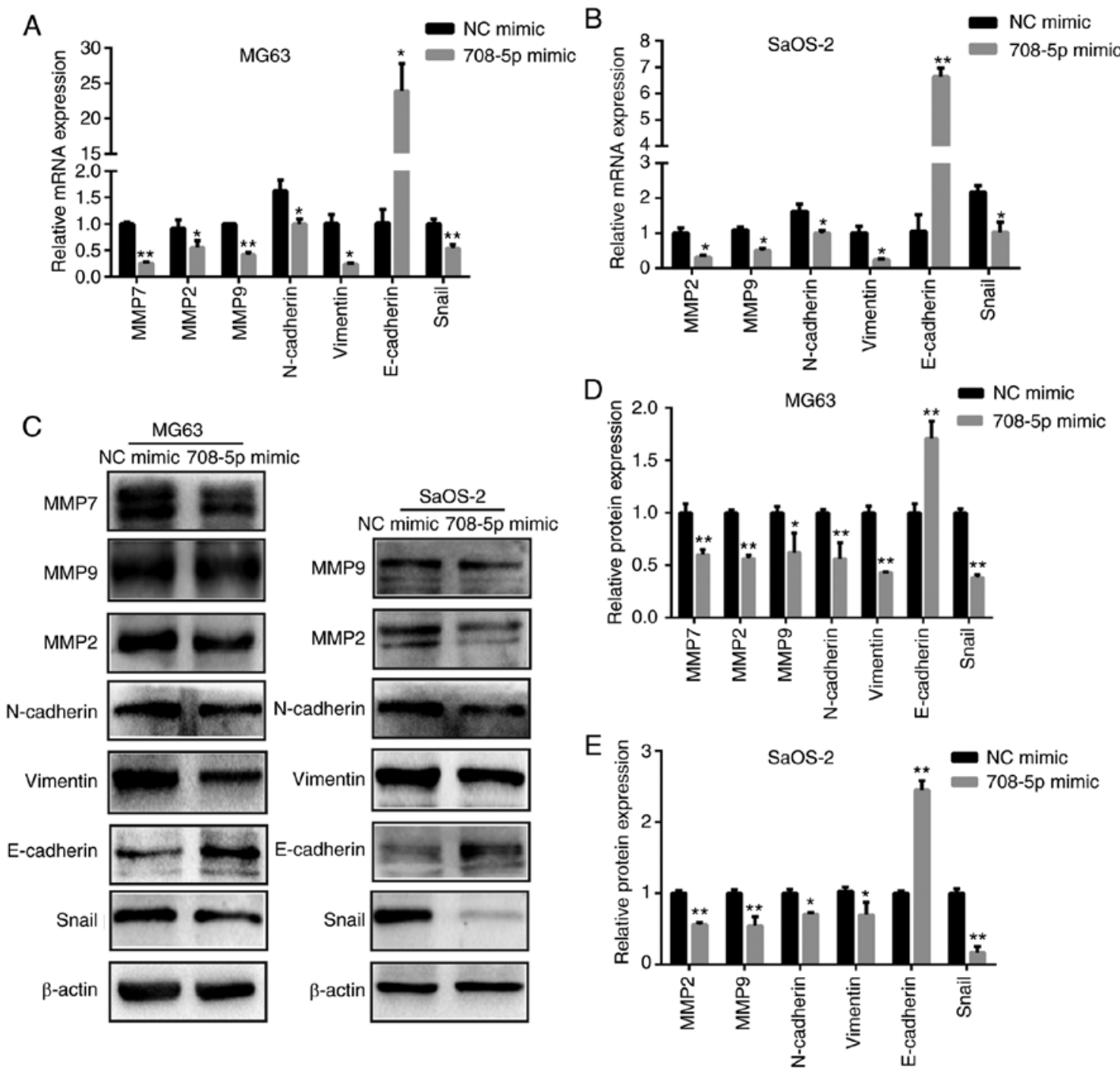

Figure 3. miR-708-5p suppresses epithelial-to-mesenchymal transition (EMT) of OS cells. (A) mRNA and (C, left column) protein levels of MMP2, MMP7, MMP9, N-cadherin, vimentin, E-cadherin and Snail after miR-708-5p overexpression (708-5p mimics) compared to the scramble negative control (NC mimic) in MG63 cells. (B) mRNA and (C, right column) protein levels of MMP2, MMP9, N-cadherin, vimentin, E-cadherin and Snail after miR-708-5p overexpression (708-5p mimics) compared to the scramble negative control (NC mimic) in SaOS-2 cells. Relative protein expression levels in (D) MG63 and (E) SaOS-2 cells. All data are presented as mean \pm SD from at least three independent experiments. ${ }^{*} \mathrm{P}<0.05,{ }^{* *} \mathrm{P}<0.01, \mathrm{NC}$ mimic vs. 708-5p mimic. OS, osteosarcoma; MMP, matrix metalloproteinase.

Except for the 3'UTR of mRNAs, 5'-UTRs or coding regions are factitiously the binding sites of miRNAs, by which miRNAs could also develop an inhibitory effect on target genes (27). Our colleagues previously illuminated the miRNA-mRNA networks through biological analysis (28), which elucidated osteosarcomagenesis-associated miRNAs and provided a theoretical basis for the discovery of the molecular mechanisms of osteosarcoma (OS) carcinogenesis.

miR-708-5p is one of the key miRNAs in numerous tumors and acts either as a tumor suppressor or promoter. miR-708-5p was recently reported to be induced by glucocorticoid receptor agonists in breast cancercells. This characteristic of miR-708-5p was found to inhibit cell proliferation, colony formation, and cell-cycle arrest (29). Not alone, miR-708-5p was suggested to be induced by glucocorticoids to impair metastasis through targeting Rap1B in ovarian cancer (30). Furthermore, suppression of miR-708-5p by polycomb group was found to increase metastasis by calcium-induced cell migration (31). Restoration of miR-708-5p expression in renal cancer cells was found to inhibit cell growth, clonability, invasion, and migration, and induce apoptosis (32). In contrast, miR-708-5p has been found to be involved in the carcinogenesis in several types of tumors. Silencing of miR-708-5p was found to promote apoptosis and inhibit growth of bladder carcinoma cells through direct repression of caspase-2 (33). In colorectal cancer, miR-708-5p was reported to be significantly upregulated when compared to normal tissues $(34,35)$. The proliferation and invasion of colorectal cancer cells were inhibited. Thus, apoptosis was found to be promoted after silencing of miR-708 (34).

In summary, miR-708-5p was found to have numerous functions in multiple types of cancers. Sui et al (36) found that miR-708-5p inhibited the growth and invasion of OS cells via regulating the URGCP/NF- $\mathrm{KB}$ signaling pathway. However, more specific effects and mechanisms that miR-708-5p may produce in OS, especially epithelial-to-mesenchymal transition (EMT), are still ambiguous. To fill this gap, we investigated 
A

\begin{tabular}{|c|cc|}
\hline miR-708-5p 3' & GGGUCGAUCUAACAUUCGAGGAA 5' \\
I I I I I I I I \\
MAP3K3 \\
3'UTR & 3'UTR WT 5' $^{\prime}$ & ..GCCCCUUUCCCUUGUAGCUCCUC..3' \\
1124-1130 & 3'UTR Mut 5' & ..GCCCCUUUCCCUUGUTCGAGCUC..3' \\
\hline
\end{tabular}

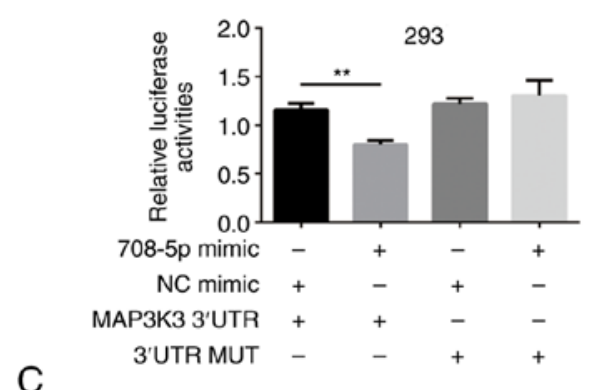

B

\begin{tabular}{|c|c|}
\hline & miR-708-5p 3' GGGUCGAUCUAACAUUCGAGGAA 5' \\
\hline $\begin{array}{c}\text { SEMA4C } \\
\text { 3'UTR }\end{array}$ & |'UTR WT 5' ..AAGCGUGGGAGGUGUAGCUCCUA..3' \\
\hline $41-48$ & 3'UTR Mut 5' ..AAGCGUGGGAGGUGUTCGAGCl \\
\hline
\end{tabular}

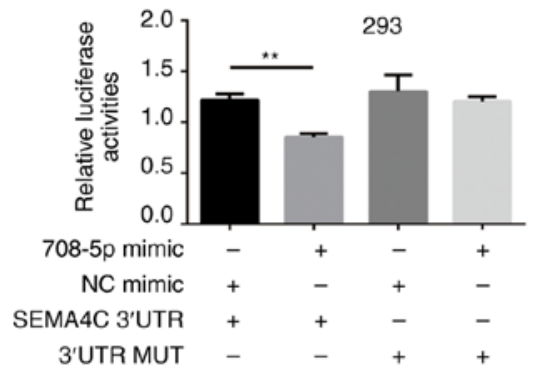

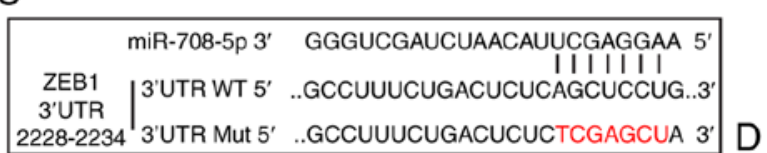

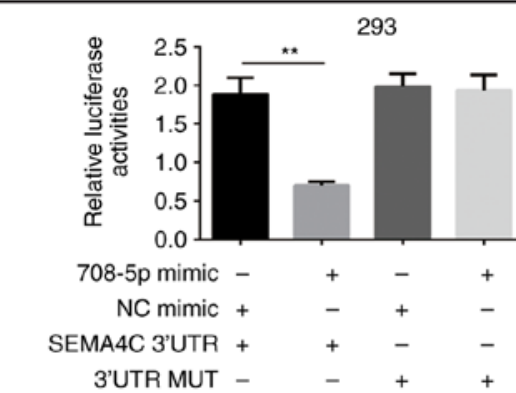

E

Hoechst 33258
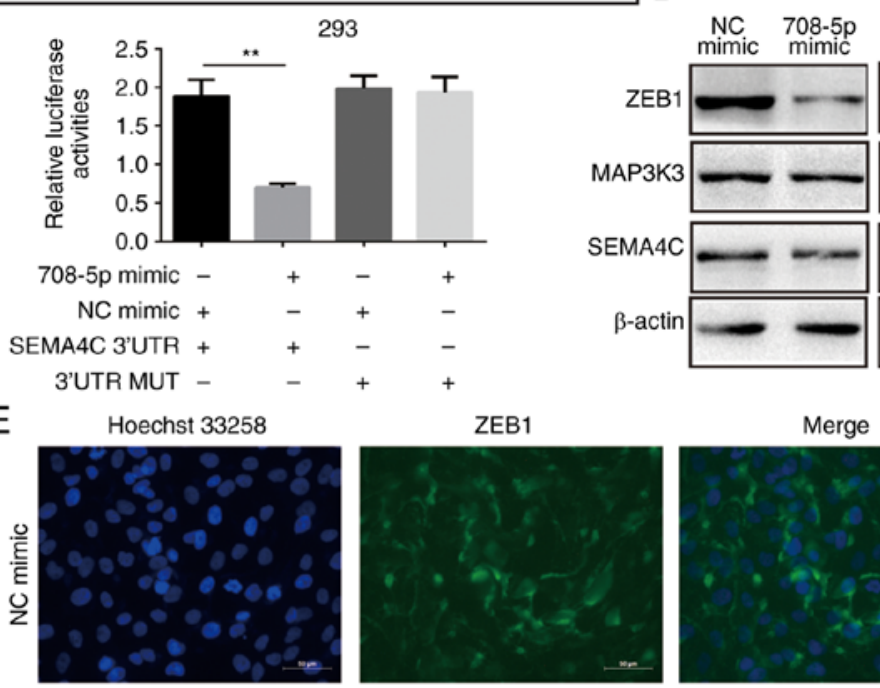

SaOS-2
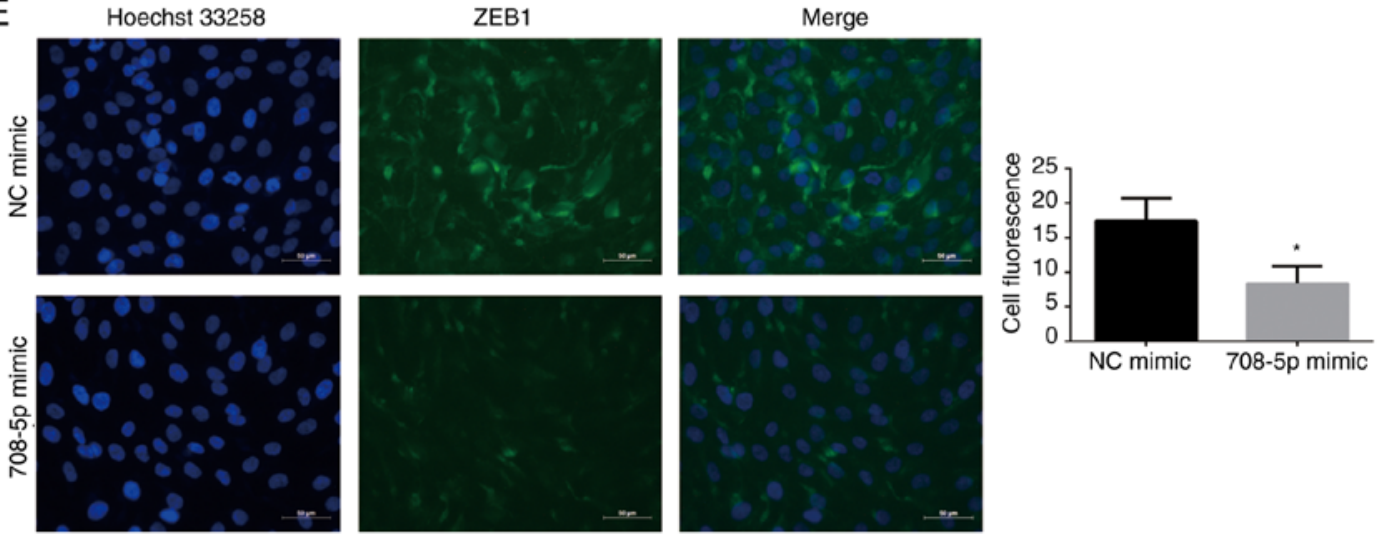

Figure 4. ZEB1 is a direct target gene of miR-708-5p. (A-C) Dual luciferase assay. (D) Western blot analysis for ZEB1, MAP3K3 and SEMA4C in MG63 and SaOS-2 cells after miR-708-5p overexpression (708-5p mimics). (E) Immunofluorescence assay for ZEB1 in MG63 cells in the 708-5p mimics group compared to the scramble negative control (NC mimic) group. All data are presented as mean $\pm \mathrm{SD}$ from at least three independent experiments. ${ }^{*} \mathrm{P}<0.05{ }^{* * *} \mathrm{P}<0.01$, NC mimic vs. 708-5p mimic. ZEB1, Zinc finger E-box-binding homeobox 1.

differentially expressed microRNAs (DEmiRNAs) in OS cells and marrow-derived mesenchymal stem cells (hMSCs). The results showed that miR-708-5p was downregulated in five OS cell lines (MG63, HOS, NY, SaOS-2, and Hu09) compared with hMSCs. Tumor-initiating cells of OS may be derived from mesenchymal stem cells in given bone circumstances (37). The differential expression of miR-708-5p in OS cells and hMSCs prompted us to consider whether it is involved in the occurrence and development of OS. Hence, we further explored the function of miR-708-5p in MG63 and SaOS-2 cells, which are two representative OS cell lines. First, we validated the downregulation of the expression of miR-708-5p in MG63 and SaOS-2 cells in comparison with two types of normal cells, namely,
hMSCs and HS-5. Then, we transfected $20 \mathrm{nM}$ of miR-708-5p mimics into OS cells, and miR-708-5p was successfully overexpressed. Restoration of miR-708-5p impaired the migration and invasion abilities of the MG63 and SaOS-2 cells. MMP2, MMP7, and MMP9 are three members of the matrix metallopeptidase gene family, which are zinc-dependent enzymes and can cleave the components of the extracellular matrix and molecules involved in signal transduction. MMPs are thought to promote cancer cell metastasis via disrupting the basement membrane and other components of ECM (38). We further discovered that the mRNA and protein levels of MMP2, MMP7, and MMP9 were significantly decreased after miR-708-5p overexpression in MG63 cells. Likewise, MMP2 

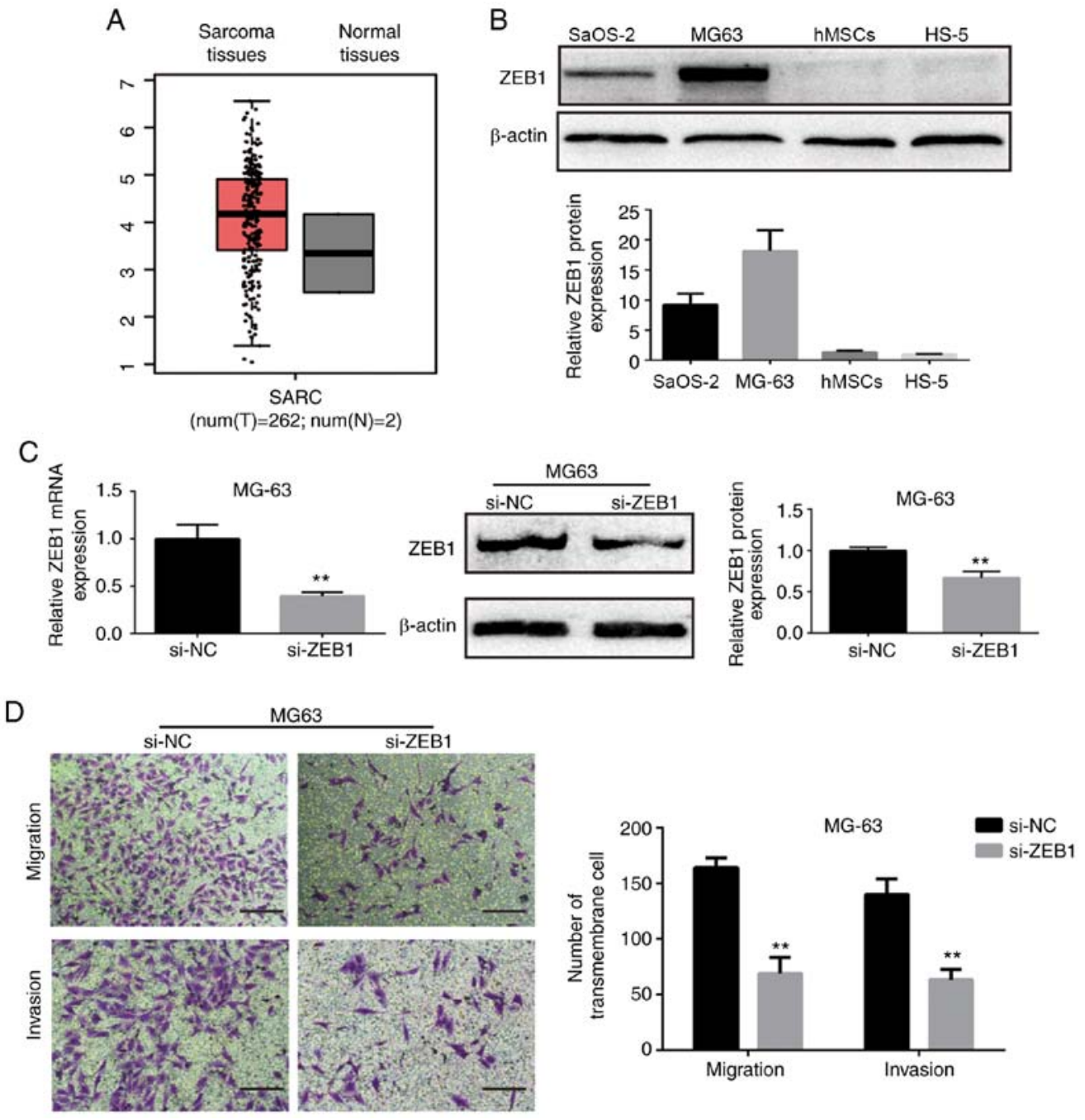

E
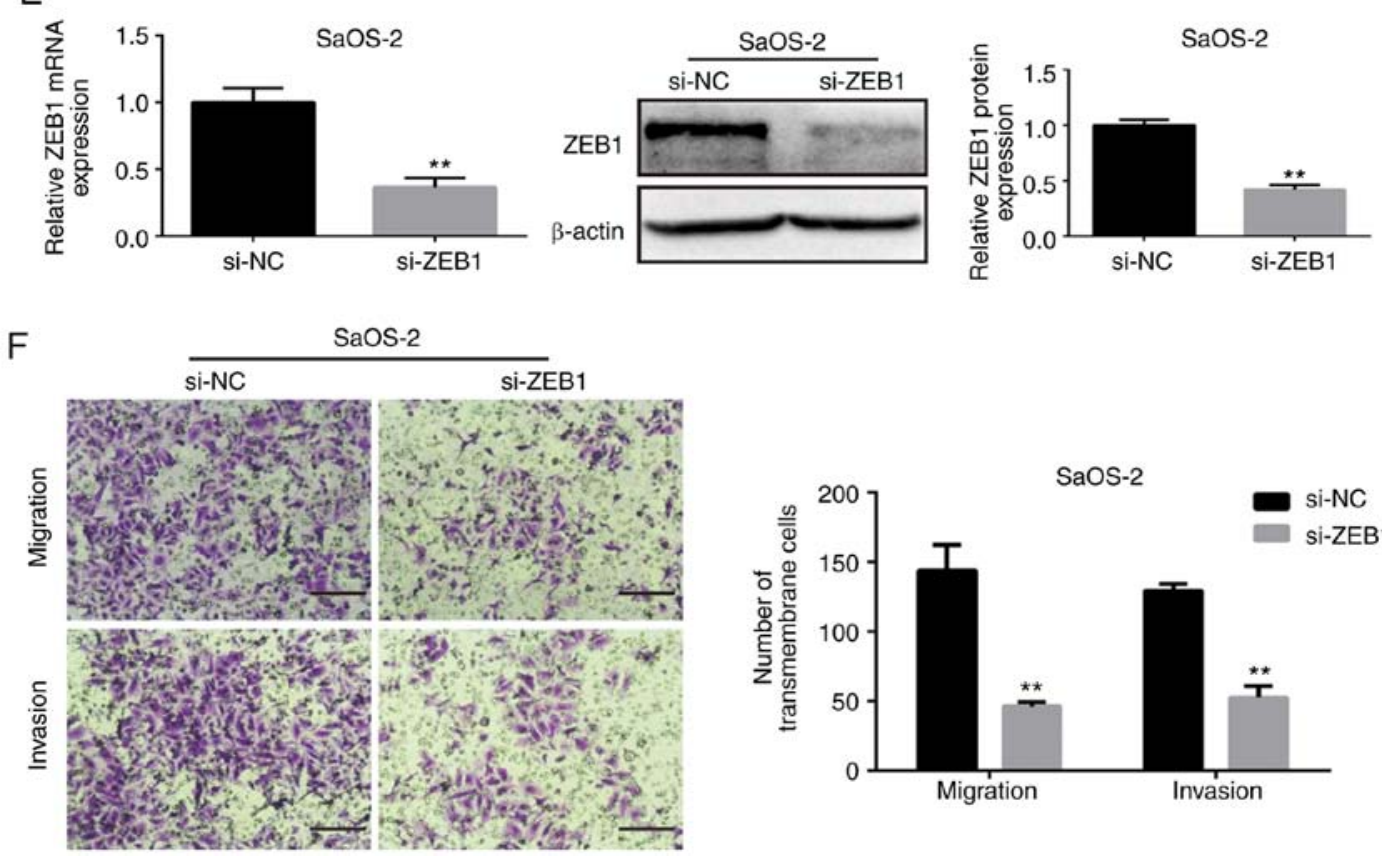

Figure 5. ZEB1 is upregulated in OS and suppression of ZEB1 inhibits OS cell migration and invasion. (A) Box plot of ZEB1 expression in sarcoma tissues from GEPIA. (B) Protein levels of ZEB1 in MG63, SaOS-2, hMSCs and HS-5 cells. (C and E) Left: mRNA levels of ZEB1 in MG63/SaOS-2 cells after si-ZEB1 transfection. Right: protein levels of ZEB1 in MG63/SaOS-2 cell after si-ZEB1 transfection. (D and F) Transwell assays for migration and invasion of MG63/SaOS-2 cells after si-ZEB1 transfection. All data are presented as mean \pm SD from at least three independent experiments. ${ }^{* * *} \mathrm{P}<0.01$, si-ZEB1 vs si-NC. OS, osteosarcoma; ZEB1, Zinc finger E-box-binding homeobox 1; hMSCs, human bone marrow-derived mesenchymal stem cells; GEPIA, Gene Expression Profiling Interactive Analysis database. 
A

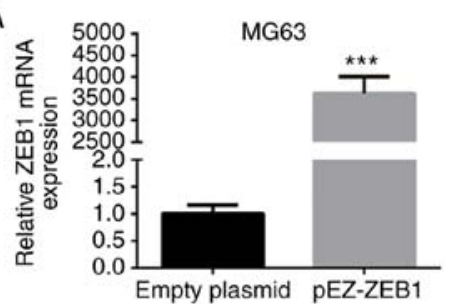

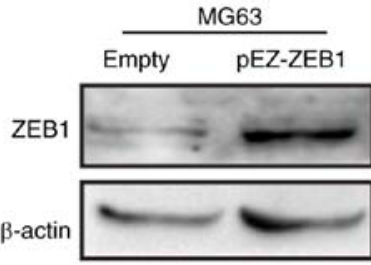

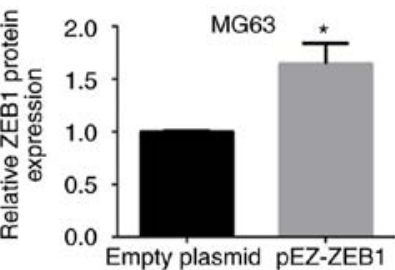

B

MG63

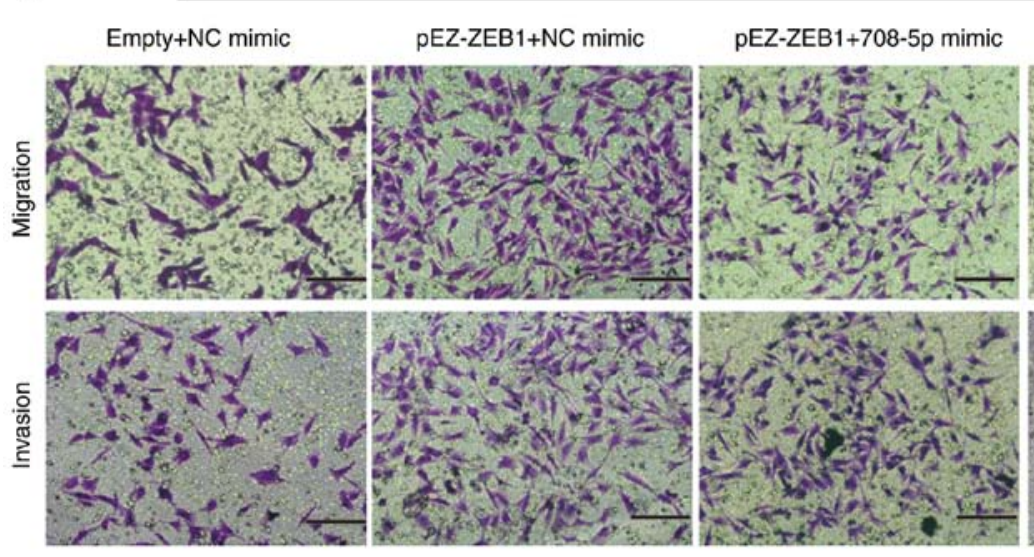

C
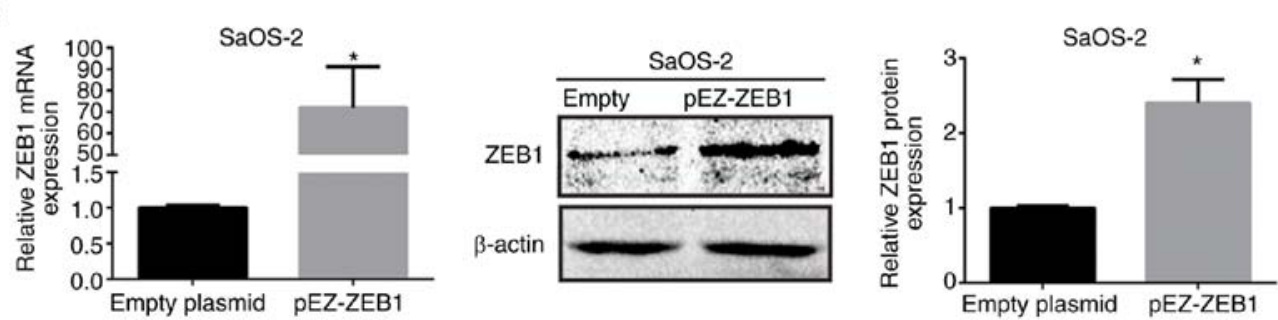

D

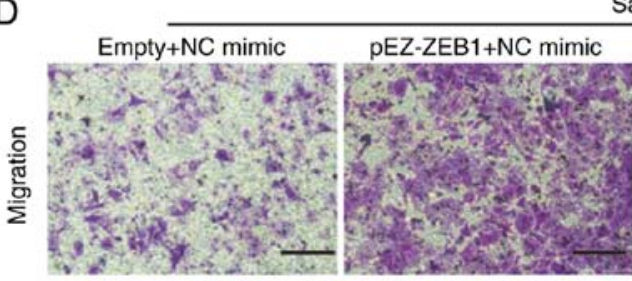

SaOS-2

\section{pEZ-ZEB1+708-5p mimic Empty+708-5p mimic}
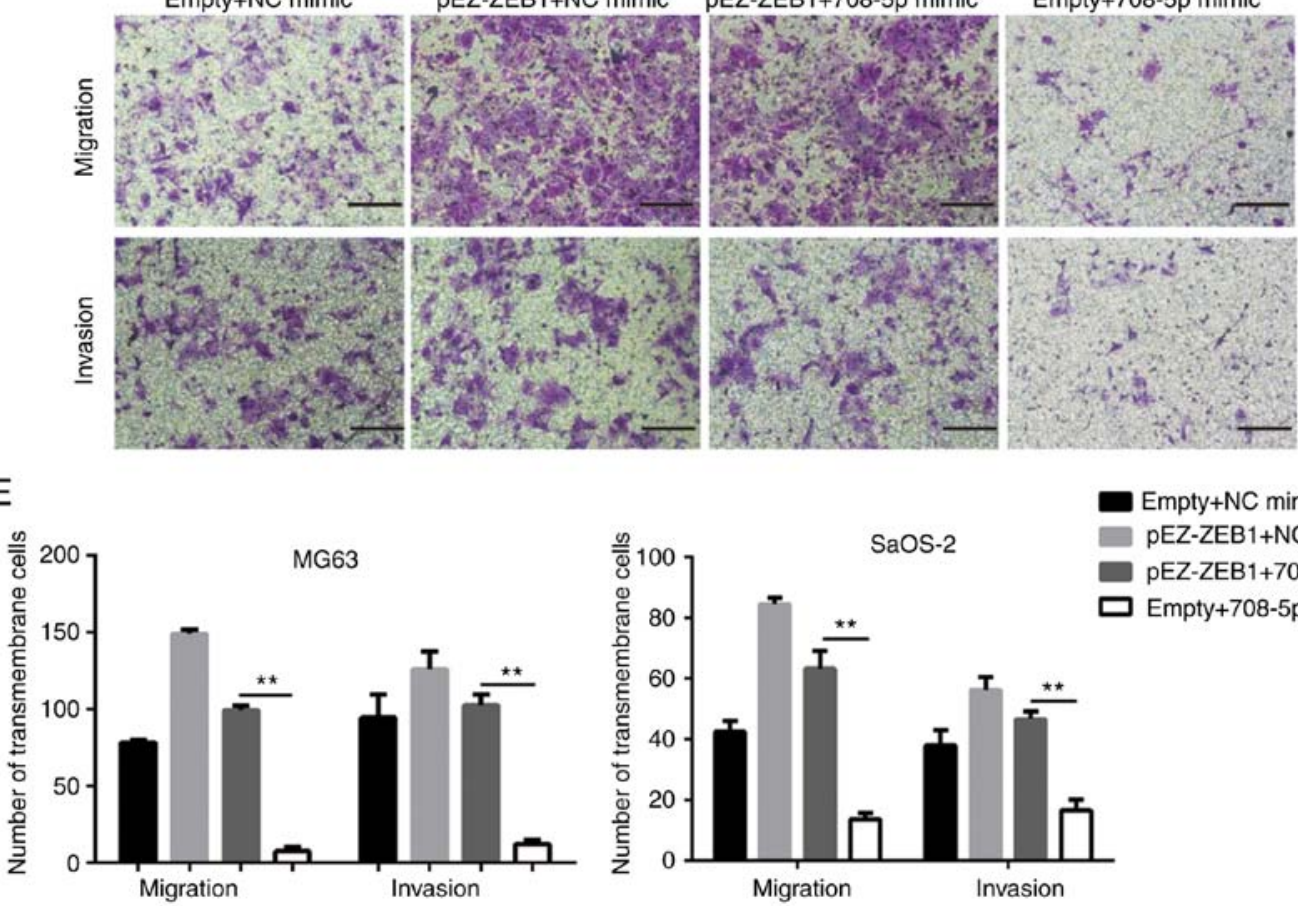

Figure 6.ZEB1 is involved in the miR-708-5p-mediated suppression of cell metastasis. (A and C) Left: mRNA levels of ZEB1 in MG63/SaOS-2 cells after transfection with the pEZ-ZEB1 plasmid. Right: protein levels of ZEB1 in MG63/SaOS-2 cells after transfection with the pEZ-ZEB1 plasmid. (B and D) Transwell assays in four groups (empty+NC mimic, pEZ-ZEB1+NC mimic, pEZ-ZEB1+708-5p mimic, pEZ-ZEB1+NC mimic) to determine MG63/SaOS-2 cell migration and invasion after transfection. (E) The numbers of migratory and invasive (transmembrane) cells in the MG63 (left) and SaOS-2 (right) cell lines. All data are presented as mean $\pm \mathrm{SD}$ from at least three independent experiments. ${ }^{*} \mathrm{P}<0.05,{ }^{* * * *} \mathrm{P}<0.001$, empty plasmid vs. pEZ-ZEB1 plasmid; ${ }^{* * *} \mathrm{P}<0.01$, pEZ-ZEB1 plasmid+708-5p mimic vs. empty+708-5p mimic. ZEB1, Zinc finger E-box-binding homeobox 1. 
and MMP9 were significantly decreased after miR-708-5p overexpression in the SaOS-2 cells.

miR-708-5p has been reported to suppress EMT in renal cancer and melanoma cells $(32,39)$. After miR-708-5p transfection, epithelial marker E-cadherin was significantly increased, whereas mesenchymal markers $\mathrm{N}$-cadherin and Vimentin were significantly decreased. Likewise, the mRNA and protein level of Snail, one of EMT-TFs, was decreased after miR-708-5p transfection. These results indicate that miR-708-5p inhibited the migration and invasion of OS through impairing EMT.

Searching and validating one or more direct target genes are essential in clarifying the specific mechanism of certain miRNAs in a given disease. We employed bioinformatics software to predict the target genes of miR-708-5p. Three tumor metastasis-associated genes, namely, $M A P 3 K 3, S E M A 4 C$, and $Z E B 1$ were chosen, as our candidate genes. Although miR-708-5p was found to bind to 3'UTR of the predicted target genes by using dual-luciferase report assay in $293 \mathrm{~T}$ cells, at the protein level, only ZEBl was significantly decreased after miR-708-5p overexpression in OS MG63 and SaOS-2 cells. Combining the results of the dual-luciferase report assay and Western blot analysis, we concluded that ZEB1 was the direct target gene of miR-708-5p in OS. To determine whether ZEB1 is involved in miR-708-5p-mediated suppression of OS cell migration and invasion, we conducted rescue experiments. We discovered that the re-expression of ZEB1 reversed the inhibition of migration and invasion mediated by miR-708-5p. This finding suggests that ZEB1 takes part in the miR-708-5p-mediated suppress of the migration and invasion of OS cells. ZEB1 is highly expressed in OS tissues when compared to normal tissue $(18,40)$. Moreover, the protein level of ZEB1 was found to be upregulated in OS MG63 and SaOS-2 cells unlike that in the hMSCs and HS-5 cells. Silencing of ZEB1 was found to inhibit the migration and invasion of MG63 and SaOS-2 cells. Thus, a miR-708-5p/ZEB1/EMT axis that mediates the metastasis of OS is established. In summary, miR-708-5p is downregulated in OS, and reinduction of the overexpression of miR-708-5p could suppress the migration and invasion abilities of OS cells by directly targeting ZEB1. Thus, miR-708-5p facilitates inhibition of EMT in OS cells. As a tumor suppressor in OS, miR-708-5p may serve as a novel diagnostic and therapeutic biomarker for OS.

\section{Acknowledgements}

Not applicable.

\section{Funding}

The present study was supported by the National Natural Science Foundation of China (grant no. 81102035).

\section{Availability of data and materials}

The datasets used and/or analyzed during the present study are available from the corresponding author on reasonable request.

\section{Authors' contributions}

TF, ZZ and GZ conceived and designed the experiments. TF performed the experiments. TF, ZZ and GZ collected, analyzed and interpreted major of the data and wrote the manuscript and provide final approval of the version to be published. YJ, HW, XM, DL, YL and LL collected, analyzed and interpreted minor of the data and revised the manuscript critically for important intellectual content. All authors read and approved the final manuscript and agree to be accountable for all aspects of the research in ensuring that the accuracy or integrity of any part of the work are appropriately investigated and resolved.

\section{Ethics approval and consent to participate}

Human bone marrow were obtained from subjects at the Children's Hospital of Chongqing Medical University (Chongqing, China) as approved by the Ethics Committee. All healthy donors provided informed consents. The study protocol was approved by the Ethics Committee of Children's Hospital of Chongqing Medical University.

\section{Patient consent for publication}

Not applicable.

\section{Competing interests}

The authors declare that they have no competing interests.

\section{References}

1. Ritter J and Bielack SS: Osteosarcoma. Ann Oncol 21 (Suppl 7): vii320-vii325, 2010

2. Smeland S, Bielack SS, Whelan J, Bernstein M, Hogendoorn P, Krailo MD, Gorlick R, Janeway KA, Ingleby FC, Anninga J, et al: Survival and prognosis with osteosarcoma: Outcomes in more than 2000 patients in the EURAMOS-1 (European and American Osteosarcoma Study) cohort. Eur J Cancer 109: 36-50, 2019.

3. Botter SM, Neri D and Fuchs B: Recent advances in osteosarcoma. Curr Opin Pharmacol 16: 15-23, 2014.

4. Meazza C and Scanagatta P: Metastatic osteosarcoma: A challenging multidisciplinary treatment. Expert Rev Anticancer Ther 16: 543-556, 2016.

5. Isakoff MS, Bielack SS, Meltzer P and Gorlick R: Osteosarcoma: Current treatment and a collaborative pathway to success. J Clin Oncol 33: 3029-3035, 2015.

6. Bartel DP: MicroRNAs: Genomics, biogenesis, mechanism, and function. Cell 116: 281-297, 2004.

7. Bartel DP: Metazoan MicroRNAs. Cell 173: 20-51, 2018.

8. Ambros V: The functions of animal microRNAs. Nature 431: 350-355, 2004.

9. Hayes J, Peruzzi PP and Lawler S: MicroRNAs in cancer: Biomarkers, functions and therapy. Trends Mol Med 20: 460-469, 2014.

10. Jones KB, Salah Z, Del Mare S, Galasso M, Gaudio E, Nuovo GJ, Lovat F, LeBlanc K, Palatini J, Randall RL, et al: miRNA signatures associate with pathogenesis and progression of osteosarcoma. Cancer Res 72: 1865-1877, 2012.

11. Delsin LEA, Roberto GM, Fedatto PF, Engel EE, Scrideli CA, Tone LG and Brassesco MS: Downregulated adhesion-associated microRNAs as prognostic predictors in childhood osteosarcoma. Pathol Oncol Res 25: 11-20, 2019.

12. Thiery JP, Acloque H, Huang RY and Nieto MA: Epithelialmesenchymal transitions in development and disease. Cell 139: 871-890, 2009.

13. Guarino M: Epithelial-mesenchymal transition and tumour invasion. Int J Biochem Cell Biol 39: 2153-2160, 2007.

14. Liao TT and Yang MH: Revisiting epithelial-mesenchymal transition in cancer metastasis: The connection between epithelial plasticity and stemness. Mol Oncol 11: 792-804, 2017.

15. Yeung KT and Yang J: Epithelial-mesenchymal transition in tumor metastasis. Mol Oncol 11: 28-39, 2017.

16. Vandewalle C, Van Roy F and Berx G: The role of the ZEB family of transcription factors in development and disease. Cell Mol Life Sci 66: 773-787, 2009. 
17. Zhang $\mathrm{Y}, \mathrm{Xu} \mathrm{L}, \mathrm{Li} \mathrm{A}$ and Han $\mathrm{X}$ : The roles of ZEB1 in tumorigenic progression and epigenetic modifications. Biomed Pharmacother 110: 400-408, 2019.

18. Shen A, Zhang Y, Yang H, Xu R and Huang G: Overexpression of ZEB1 relates to metastasis and invasion in osteosarcoma. J Surg Oncol 105: 830-834, 2012.

19. Jiang R, Zhang C, Liu G, Gu R and Wu H: MicroRNA-126 inhibits proliferation, migration, invasion and EMT in osteosarcoma by targeting ZEB1. J Cell Biochem 118: 3765-3774, 2017.

20. Yi L, Liu M and Tang Z: MicroRNA-130a inhibits growth and metastasis of osteosarcoma cells by directly targeting ZEB1. Mol Med Rep 16: 3606-3612, 2017.

21. Deng Y,Luan F,Zeng L, Zhang Y and Ma K: MiR-429 suppresses the progression and metastasis of osteosarcoma by targeting ZEB1. EXCLI J 16: 618-627, 2017.

22. Strong AL, Jiang Q, Zhang Q, Zheng S, Boue SM, Elliott S Burow ME, Bunnell BA and Wang G: Design, synthesis, and osteogenic activity of daidzein analogs on human mesenchymal stem cells. ACS Med Chem Lett 5: 143-148, 2013.

23. Zhou Y, Jiang R, An L, Wang H, Cheng S, Qiong S and Weng Y: Benzo[a]pyrene impedes self-renewal and differentiation of mesenchymal stem cells and influences fracture healing. Sci Total Environ 587-588: 305-315, 2017.

24. Livak KJ and Schmittgen TD: Analysis of relative gene expression data using real-time quantitative PCR and the 2-(-Delta Delta C (T)) method. Methods 25: 402-408, 2001.

25. Friedman RC, Farh KK, Burge CB and Bartel DP: Most mammalian mRNAs are conserved targets of microRNAs. Genome Res 19: 92-105, 2009

26. Filipowicz W, Bhattacharyya SN and Sonenberg N: Mechanisms of post-transcriptional regulation by microRNAs: Are the answers in sight? Nat Rev Genet 9: 102-114, 2008.

27. Lytle JR, Yario TA and Steitz JA: Target mRNAs are repressed as efficiently by microRNA-binding sites in the 5' UTR as in the 3' UTR. Proc Natl Acad Sci USA 104: 9667-9672, 2007.

28. Wang H, Tang M, Ou L, Hou M, Feng T, Huang YE, Jin Y,Zhang H and Zuo G: Biological analysis of cancer specific microRNAs on function modeling in osteosarcoma. Sci Rep 7: 5382, 2017.

29. Senthil Kumar KJ, Gokila Vani M, Hsieh HW, Lin CC, Liao JW, Chueh PJ and Wang SY: MicroRNA-708 activation by glucocorticoid receptor agonists regulate breast cancer tumorigenesis and metastasis via downregulation of NF- $\mathrm{KB}$ signaling. Carcinogenesis 40: 335-348, 2019.

30. Lin KT, Yeh YM, Chuang CM, Yang SY, Chang JW, Sun SP, Wang YS, Chao KC and Wang LH: Glucocorticoids mediate induction of microRNA-708 to suppress ovarian cancer metastasis through targeting Rap1B. Nat Commun 6: 5917, 2015.
31. Ryu S, McDonnell K, Choi H, Gao D, Hahn M, Joshi N, Park SM, Catena R, Do Y, Brazin J, et al: Suppression of miRNA-708 by polycomb group promotes metastases by calcium-induced cell migration. Cancer Cell 23: 63-76, 2013.

32. Saini S, Yamamura S, Majid S, Shahryari V, Hirata H, Tanaka Y and Dahiya R: MicroRNA-708 induces apoptosis and suppresses tumorigenicity in renal cancer cells. Cancer Res 71: 6208-6219, 2011.

33. Song T, Zhang X, Zhang L, Dong J, Cai W, Gao J and Hong B: miR-708 promotes the development of bladder carcinoma via direct repression of Caspase-2. J Cancer Res Clin Oncol 139: 1189-1198, 2013.

34. Lei SL, Zhao H, Yao HL, Chen Y, Lei ZD, Liu KJ and Yang Q: Regulatory roles of microRNA-708 and microRNA-31 in proliferation, apoptosis and invasion of colorectal cancer cells. Oncol Lett 8: 1768-1774, 2014

35. Piepoli A, Tavano F, Copetti M, Mazza T, Palumbo O, Panza A, di Mola FF, Pazienza V, Mazzoccoli G, Biscaglia G, et al: MiRNA expression profiles identify drivers in colorectal and pancreatic cancers. PLoS One 7: e33663, 2012.

36. Sui C, Liu D, Hu Y and Zhang L: MicroRNA-708-5p affects proliferation and invasion of osteosarcoma cells by targeting URGCP. Exp Ther Med 17: 2235-2241, 2019.

37. Gambera S, Abarrategi A, Rodríguez-Milla MA, Mulero F, Menéndez ST, Rodriguez R, Navarro S and García-Castro J: Role of activator protein-1 complex on the phenotype of human osteosarcomas generated from mesenchymal stem cells. Stem Cells 36: 1487-1500, 2018.

38. Yoon SO, Park SJ, Yun CH and Chung AS: Roles of matrix metalloproteinases in tumor metastasis and angiogenesis. J Biochem Mol Biol 36: 128-137, 2003.

39. Song XF, Wang QH and Huo R: Effects of microRNA-708 on epithelial-mesenchymal transition, cell proliferation and apoptosis in melanoma cells by targeting LEF1 through the Wnt signaling pathway. Pathol Oncol Res 25: 377-389, 2019.

40. Yan H, Zhang B, Fang C and Chen L: miR-340 alleviates chemoresistance of osteosarcoma cells by targeting ZEB1. Anticancer Drugs 29: 440-448, 2018.

This work is licensed under a Creative Commons Attribution-NonCommercial-NoDerivatives 4.0 International (CC BY-NC-ND 4.0) License. 